\title{
Atividade física e qualidade de vida de professores universitários
}

\section{Physical activity and life quality of university professors}

\author{
Samuel Carvalho Dumith ${ }^{1}$ (D) \\ 'Programa de Pós-graduação em Saúde Pública, Faculdade de Medicina, Universidade Federal do Rio Grande (FURG), Rio Grande (RS), Brasil.
}

Como citar: Dumith SC. Atividade física e qualidade de vida de professores universitários. Cad Saúde Colet, 2020;28(3):438-446. https://doi.org/10.1590/1414-462X202028030593

\section{Resumo}

Introdução: A qualidade de vida é um tema que tem sido investigado em diversas áreas do conhecimento e sob diversos enfoques. Objetivo: Investigar a prática de atividade física de lazer em professores universitários e analisar sua associação com a qualidade de vida. Método: A população-alvo foram os professores da Universidade Federal do Rio Grande, RS, Brasil, que estavam em atividade e atuavam nos campi da cidade de Rio Grande no segundo semestre de 2015. A coleta se deu por meio do preenchimento de um questionário eletrônico. A qualidade de vida foi avaliada pelo instrumento The Medical Outcomes Study 36-item Short Form Health Survey (SF-36). Os dados foram analisados mediante análise de variância ou teste não paramétrico de Kruskal-Wallis. Resultados: A amostra contou com 270 participantes, correspondendo a $40 \%$ dos professores elegíveis. Relataram praticar alguma atividade física no tempo livre $70,4 \%$ dos professores, sendo que $43,0 \%$ (IC95\% 37,0 a 48,9) foram considerados fisicamente ativos no lazer ( $\geq 150 \mathrm{~min} / \mathrm{sem}$ ). Este grupo obteve maior escore de qualidade de vida, sendo verificada associação linear positiva entre atividade física e o escore de qualidade de vida $(p<0,01)$. Conclusão: Menos da metade dos professores universitários foi considerada fisicamente ativa. Observou-se associação linear positiva entre prática de atividade física no lazer com maior qualidade de vida.

Palavras-chave: qualidade de vida; atividade motora; docentes; estilo de vida; epidemiologia.

\begin{abstract}
Background:The quality of life is a subject that has been investigated in several areas of knowledge and under many approaches. Objective: To investigate the practice of leisure time physical activity among university professors and to analyze its association with quality of life. Method: The target population was professors of the Federal University of the Rio Grande, Brazil, who were active and worked on campus in the city of the Rio Grande in the second half of 2015. The collection was carried out by completing an electronic questionnaire. The quality of life was assessed by the instrument The Medical Outcomes Study 36- item Short Form Health Survey (SF-36). Data were analyzed by analysis of variance test or nonparametric Kruskal-Wallis test. Results: The sample included 270 participants, representing $40 \%$ of eligible professors. There were $70.4 \%$ that reported performing some physical activity in leisure time, and $43.0 \%(95 \% \mathrm{Cl} 37.0$ to 48.9$)$ were considered physically active ( $\geq 150 \mathrm{~min} /$ week). This group obtained the highest score for quality of life, suggesting a linear positive association between physical activity and the score of quality of life $(p<0.01)$. Conclusions: Less than half of college professors were considered physically active. There was a positive linear association of physical activity practice with higher scores of quality of life.
\end{abstract}

Keywords: quality of life; motor activity; professors; life style; epidemiology.

\section{(c) (i)}

Este é um artigo publicado em acesso aberto (Open Access) sob a licença Creative Commons Attribution, que permite uso, distribuição e reprodução em qualquer meio, sem restrições desde que o trabalho original seja corretamente citado. 


\section{INTRODUÇÃO}

A qualidade de vida é um tema que tem sido investigado em diversas áreas do conhecimento e sob diversos enfoques. Estudo de revisão sistemática apontou que o número de artigos sobre qualidade de vida, como descritor no portal SciELO, aumentou de sete em 2001 para 54 em 2006'. Esse dado demonstra que este tema vem ganhando grande importância no campo acadêmico-científico.

Acredita-se que o estilo de vida exerça influência sobre a qualidade de vida ${ }^{2}$. Estudos internacionais evidenciaram que existe uma associação positiva entre qualidade de vida e atividade física ${ }^{3}$. Em 2010, foi realizada uma revisão sistemática, que abrangeu 38 publicações sobre a relação entre atividade física e qualidade de vida, e constatou-se que a maioria desses estudos foram conduzidos com idosos ou com portadores de alguma doença/enfermidade ${ }^{4}$. Apenas nove deles foram conduzidos com adultos aparentemente saudáveis. Aproximadamente quatro quintos (81\%) destes estudos foram conduzidos na América do Norte (42\%), Europa (21\%) e Ásia (18\%). O instrumento mais utilizado para avaliar qualidade de vida foi o The Medical Outcomes Study 36-item Short Form Health Survey (SF-36), que apareceu em 27 publicações (71\% do total) $)^{4}$.

Um público, em particular, que merece especial atenção, devido a sua característica peculiar de potenciais formadores de opinião e de profissionais capacitados, são os professores. Em 2015, foi publicada uma revisão sistemática sobre estudos brasileiros que avaliaram a qualidade de vida em professores entre 2000 e $2014^{5}$. Neste estudo, os autores encontraram 12 artigos, sendo que sete deles foram feitos com professores do ensino superior ${ }^{5}$. Houve preponderância de estudos quantitativos, transversais, com instrumentos padronizados, e uma maior concentração de artigos publicados a partir de $2012^{5}$.

Ainda existem poucos estudos no Brasil que tenham traçado as características dos professores fisicamente ativos, e menos estudos ainda que tenham relacionado o estilo de vida ativo com a qualidade de vida em docentes. Sendo assim, conhecer quais são as características dos professores fisicamente inativos poderá contribuir para intervir sobre tais grupos, mesmo que no âmbito universitário. Ainda que seja esperada uma associação positiva do nível de atividade física com a qualidade de vida, a confirmação deste achado poderá trazer mais uma evidência sobre os benefícios de se ter um estilo de vida fisicamente ativo, e uma motivação a mais para os docentes inativos começarem a praticar atividade física.

Em vista disso, o presente artigo foi concebido e delineado com os seguintes objetivos: i) investigar a prática de atividade física e os fatores associados em professores universitários; ii) analisar a associação da prática de atividade física com a qualidade de vida destes professores.

\section{MÉTODO}

Este estudo teve caráter observacional do tipo transversal com abordagem quantitativa. Foi aprovado pelo Comitê de Ética em Pesquisa na Área da Saúde (CEPAS) da Universidade Federal do Rio Grande (FURG) sob número 72/2015 (CAAE: 48819115.1.0000.5324). Todos os professores da FURG que estavam em atividade e atuavam nos campi da cidade de Rio Grande (Campus Carreiros e Campus Saúde) no segundo semestre de 2015 foram convidados a participar da pesquisa.

A coleta dos dados ocorreu durante os meses de outubro e novembro de 2015. Todos os professores elegíveis receberam por e-mail um convite para participar da pesquisa. Neste mesmo e-mail constava o link que remetia ao questionário empregado na pesquisa. Este questionário foi gerado pelo Google Docs e deveria ser preenchido no próprio computador, bastando ter conexão com internet para abrir e enviar. Antes de responder o questionário, os professores deveriam assinalar que consentiam em fazer parte do estudo. Não foi coletada nenhuma informação referente a nome, nem número de matrícula, sendo as respostas anônimas.

A qualidade de vida dos indivíduos foi analisada por meio do questionário denominado SF-36, o qual é um questionário padronizado, genérico de avaliação de qualidade de vida, de fácil entendimento e aplicabilidade. Ele é formado por 36 itens, englobados em oito domínios: 
capacidade funcional, aspectos físicos, dor, estado geral da saúde, vitalidade, aspectos sociais, aspectos emocionais e saúde mental. Apresenta um escore final de 0 a 100, em que o zero corresponde ao pior estado geral de saúde e 100 equivale ao melhor estado de saúde, podendo ser aplicado de forma autoadministrada, informatizada, por entrevista ou por telefone ${ }^{6}$. Foi traduzido pelo método de back translation em 1999 e é aplicado em diversas pesquisas, abrangendo múltiplas populações, em condições distintas ${ }^{7}$. Neste estudo, o coeficiente alfa de Cronbach para as 36 questões do SF-36 foi de 0,92, indicando consistência interna excelente ${ }^{8}$.

O nível de atividade física de lazer referiu-se a uma semana habitual e foi adaptado do questionário do inquérito denominado Vigilância de Fatores de Risco e Proteção para Doenças Crônicas por Inquérito Telefônico (VIGITEL) ${ }^{9}$. Os professores foram questionados acerca da frequência (em dias) e do tempo diário (em minutos) destas atividades. Assim, multiplicando-se a frequência semanal pelo tempo diário, obteve-se a quantidade total de atividade física no lazer em minutos por semana ( $\mathrm{min} / \mathrm{sem}$ ). Aqueles que atingiram $150 \mathrm{~min} / \mathrm{sem}$ ou mais foram classificados como fisicamente ativos ${ }^{10}$. O nível de atividade física (em $\mathrm{min} / \mathrm{sem}$ ) foi também dividido em tercis e quartis, a fim de permitir testar se havia um gradiente de dose resposta com a qualidade de vida.

As demais variáveis foram: sexo (masculino e feminino); faixa etária (em décadas); estado civil (casado e não casado); instituto em que trabalha (Saúde e outros); tempo de trabalho na instituição (<5 anos, 5 a 9, 10 a 19 e 20 ou mais); doutorado (não e sim); contrato com dedicação exclusiva (não e sim); tabagismo (não fumante, fumante e ex-fumante); hábito de tomar café da manhã (não e sim); consumo diário de frutas (não e sim); consumo de refrigerante (não e sim); estado nutricional, mensurado pelo índice de massa corporal (IMC) a partir do peso e estatura autorreferidos (eutrófico: IMC $<25 \mathrm{~kg} / \mathrm{m}^{2}$, sobrepeso: IMC entre 25,0 e $29,9 \mathrm{~kg} / \mathrm{m}^{2}$ e obeso: IMC $\geq 30 \mathrm{~kg} / \mathrm{m}^{2}$ ); diagnóstico médico autorreferido de hipertensão arterial (não ou sim); autopercepção da saúde (excelente ou muito boa, boa, regular ou ruim). Todas as opções de respostas às perguntas foram fechadas, devendo o respondente assinalar uma das opções de resposta.

Os dados foram exportados para um arquivo em Excel, que posteriormente foi transferido para o pacote estatístico Stata, versão 11.1, e realizada a limpeza do banco de dados, criação das variáveis e análise dos dados. Para a descrição dos dados, empregou-se frequência absoluta (N) e relativa (\%). A análise bivariada entre a prevalência de professores fisicamente ativos no lazer com as demais variáveis foi conduzida pelo teste exato de Fisher. Para a análise bruta e ajustada, usou-se regressão de Poisson com ajuste robusto para variância para estimar as Razões de Prevalências (RP) e os Intervalos de Confiança a 95\% (IC95\%) ${ }^{11}$.

A análise ajustada foi realizada seguindo um modelo de análise em níveis com regressão para trás, isto é, as variáveis eram controladas para aquelas do mesmo nível ou de níveis acima ${ }^{12}$. No primeiro nível, entraram sexo, faixa etária e estado civil; no segundo, instituto, tempo de trabalho, titulação de doutorado e contrato com dedicação exclusiva; no terceiro, foram incluídos tabagismo, hábito de tomar café da manhã, consumo de frutas e consumo de refrigerante; e no quarto nível, colocou-se estado nutricional, hipertensão arterial e autopercepção da saúde. Foram mantidas no modelo final as variáveis que obtiveram valor $p<0,20$ na análise ajustada.

Procedeu-se a uma análise de componentes principais ${ }^{13} \mathrm{com}$ o intuito de extrair uma única variável de qualidade de vida a partir do escore obtido em cada um dos oito domínios. Nessa análise, extraiu-se o primeiro componente, que explicou $48 \%$ da variabilidade de todos os componentes (Eigenvalue $=3,9$ ). Esse componente de qualidade de vida foi gerado em escore-z, com média teórica igual a zero e desvio-padrão igual a um. Essa variável foi analisada na sua forma contínua (em escore-z) por meio da regressão linear simples e múltipla. A interpretação é de que, quanto maior o escore-z, maior o nível de qualidade de vida. Tal variável foi comparada com a atividade física, na sua forma dicotômica, em tercis e em quartis. Na análise ajustada do escore-z de qualidade de vida com atividade física, controlou-se para aquelas variáveis que tiveram valor $\mathrm{p}<0,20$ na associação com atividade física.

A análise da associação entre atividade física (em tercis) e os domínios de qualidade de vida foi testada pela análise de variância (ANOVA), com teste post-hoc de Bonferroni, ou teste 
de Kruskal-Wallis, quando o teste de homogeneidade das variâncias de Bartlett's atingia valor $\mathrm{p}<0,10$. O nível de significância estatístico utilizado foi de $5 \%$ para testes bicaudais, e para manutenção das variáveis no modelo ajustado empregou-se o valor $p$ de 0,20 . Para variáveis categóricas ordinais, reportou-se o valor $p$ do teste de tendência linear.

\section{RESULTADOS}

De um total de 680 professores atuantes nos campi da FURG em Rio Grande, RS, a amostra contou com 270 participantes, correspondendo a $40 \%$ dos professores elegíveis. Destes, pouco mais da metade $(52,2 \%)$ foi do sexo feminino, um quarto $(25,9 \%)$ tinha 50 anos ou mais, dois terços (65,0\%) eram casados, $11,5 \%$ estavam lotados no campus Saúde, um terço (32,6\%) estava há menos de cinco anos na instituição, três quartos $(75,0 \%)$ possuíam doutorado, $84,1 \%$ tinham dedicação exclusiva, $6,3 \%$ foram fumantes, $14,8 \%$ não tomavam café da manhã regularmente, $63,0 \%$ não consumiam frutas diariamente, $60,7 \%$ consumiam refrigerante, quase metade $(48,2 \%)$ estava acima do peso (sobrepeso ou obesidade), $17,4 \%$ referiram diagnóstico médico de hipertensão arterial e 13\% percebiam sua saúde como regular ou ruim.

Relataram praticar alguma atividade física no tempo livre 70,4\% dos professores, sendo que $43,0 \%$ (IC95\% 37,0 a 48,9) foram considerados fisicamente ativos ( $\geq 150 \mathrm{~min} / \mathrm{sem}$ ). A mediana de atividade física de lazer foi de $90 \mathrm{~min} / \mathrm{sem}$, variando de 0 a $420 \mathrm{~min} / \mathrm{sem}$. A prevalência de atividade física no lazer oscilou de 20,0\%, para aqueles que não costumavam tomar café da manhã, até $61,7 \%$, para aqueles que referiram diagnóstico médico de hipertensão arterial.

Na Tabela 1 são apresentadas a análise bruta e ajustada da atividade física no lazer e as características estudadas dos professores. Percebe-se que houve uma associação diretamente proporcional com a faixa etária, ou seja, quanto maior a idade, maior foi a prevalência de indivíduos fisicamente ativos no lazer $(p<0,01)$. 0 tempo de trabalho na instituição, que também teve uma associação diretamente proporcional com atividade física na análise bruta $(p=0,02)$, perdeu associação na análise ajustada $(p=0,60)$ devido ao efeito confundidor imposto pela idade do professor. Possuir título de doutor ficou no limiar da significância estatística $(p=0,05)$ após ajuste para possíveis fatores de confusão. Os ex-fumantes foram mais ativos que o grupo não fumante na análise bruta $(p=0,03)$, perdendo a associação na análise ajustada $(p=0,09)$. Tomar café da manhã regularmente e consumir frutas diariamente se mantiveram associados com maior prevalência de atividade física. Professores que não consumiam refrigerantes foram mais ativos na análise bruta $(p=0,02)$, com perda de significância após controle para confusão $(p=0,60)$. Professores com sobrepeso e com hipertensão arterial foram mais ativos fisicamente do que seus pares. A autopercepção da saúde teve uma associação positiva com atividade física na análise bruta $(p=0,03)$, perdendo a associação na ajustada $(p=0,09)$.

$\mathrm{Na}$ análise entre atividade física e qualidade de vida (Tabela 2), verificou-se que professores fisicamente ativos ( $\geq 150 \mathrm{~min} / \mathrm{sem}$ ) tiveram maior escore de qualidade de vida, tanto na análise bruta, quanto na ajustada. Foi observada também um associação linear positiva entre atividade física em tercis e em quartis e escore de qualidade de vida $(p<0,01)$. Salienta-se que as maiores diferenças foram encontradas entre os grupos extremos (menos e mais ativos fisicamente).

Na Tabela 3 é mostrada a associação da atividade física (em tercis) com os escores de qualidade de vida para cada domínio. Nota-se que, para os seguintes domínios: capacidade funcional, estado geral de saúde, vitalidade e saúde mental, houve uma associação linear positiva com o nível de atividade física (todos com valor $p$ de tendência $<0,01$ ). No domínio capacidade funcional, houve diferença entre o grupo mais ativo e o menos ativo $(p<0,01)$. Nos domínios vitalidade $(p<0,01)$ e saúde mental $(p=0,01)$, a diferença encontrada foi para o grupo mais ativo versus os demais. No domínio estado geral de saúde, houve diferença estatisticamente significativa entre os três grupos $(p<0,01)$. Já no domínio aspectos físicos, verificou-se uma associação limítrofe, sendo que o grupo menos ativo apresentou a maior pontuação. Os domínios dor, aspectos sociais e aspectos emocionais não demonstraram associação com o nível de atividade física. 
Tabela 1. Análise da prática de atividade física de lazer ( $\geq 150 \mathrm{~min} / \mathrm{sem}$ ) com características dos professores da Universidade Federal do Rio Grande (FURG), Rio Grande, RS, 2015 (N=270)

\begin{tabular}{|c|c|c|c|c|c|}
\hline \multirow{2}{*}{ Variável } & \multirow{2}{*}{$\begin{array}{c}\% \\
\text { ativos }\end{array}$} & \multicolumn{2}{|c|}{ Análise bruta } & \multicolumn{2}{|c|}{ Análise ajustada } \\
\hline & & RP (IC95\%) & Valor $p$ & RP (IC95\%) & Valor $p$ \\
\hline Sexo & & & 0,53 & & 0,71 \\
\hline Masculino & 45,0 & $1,09(0,83-1,44)$ & & $1,05(0,80-1,39)$ & \\
\hline Feminino & 41,1 & 1,00 & & 1,00 & \\
\hline Faixa etária & & & $<0,01^{*}$ & & $<0,01^{*}$ \\
\hline 20-29 anos & 24,0 & $0,40(0,18-0,90)$ & & $0,40(0,18-0,90)$ & \\
\hline 30-39 anos & 37,5 & $0,63(0,38-1,02)$ & & $0,63(0,38-1,02)$ & \\
\hline 40-49 anos & 46,8 & $0,78(0,49-1,26)$ & & $0,78(0,49-1,26)$ & \\
\hline 50-59 anos & 50,9 & $0,85(0,52-1,38)$ & & $0,85(0,52-1,38)$ & \\
\hline 60-69 anos & 60,0 & 1,00 & & 1,00 & \\
\hline Estado civil & & & 0,34 & & 0,93 \\
\hline Casado(a) & 45,1 & 1,00 & & 1,00 & \\
\hline $\begin{array}{l}\text { Solteiro(a), Divorciado(a), } \\
\text { Viúvo(a) }\end{array}$ & 39,0 & $0,86(0,64-1,17)$ & & $0,99(0,73-1,33)$ & \\
\hline Instituto & & & 0,85 & & 0,64 \\
\hline $\begin{array}{l}\text { Saúde (enfermagem e } \\
\text { medicina) }\end{array}$ & 45,2 & $0,95(0,62-1,43)$ & & $1,12(0,70-1,78)$ & \\
\hline Outros & 42,7 & 1,00 & & 1,00 & \\
\hline $\begin{array}{l}\text { Tempo de trabalho na } \\
\text { instituição }\end{array}$ & & & $0,02^{*}$ & & $0,60^{*}$ \\
\hline$<5$ anos & 33,0 & 1,00 & & 1,00 & \\
\hline 5 a 9 anos & 45,2 & $1,37(0,94-2,00)$ & & $1,17(0,79-1,73)$ & \\
\hline 10 a 19 anos & 48,7 & $1,48(0,96-2,29)$ & & $1,14(0,69-1,88)$ & \\
\hline$\geq 20$ anos & 51,8 & $1,57(1,06-2,32)$ & & $1,16(0,68-1,98)$ & \\
\hline Possui doutorado & & & 0,03 & & 0,05 \\
\hline Não & 30,9 & 1,00 & & 1,00 & \\
\hline Sim & 47,0 & $1,52(1,04-2,24)$ & & $1,60(0,99-2,59)$ & \\
\hline Dedicação exclusiva & & & 0,43 & & 0,16 \\
\hline Não & 37,2 & 1,00 & & 1,00 & \\
\hline Sim & 44,1 & $1,18(0,78-1,79)$ & & $0,68(0,40-1,17)$ & \\
\hline Tabagismo & & & 0,03 & & 0,09 \\
\hline Não fumante & 40,2 & 1,00 & & 1,00 & \\
\hline Fumante & 23,5 & $0,59(0,25-1,41)$ & & $0,63(0,26-1,53)$ & \\
\hline Ex-fumante & 55,1 & $1,37(1,04-1,81)$ & & $1,28(0,98-1,69)$ & \\
\hline $\begin{array}{l}\text { Toma café da manhã } \\
\text { regularmente }\end{array}$ & & & 0,01 & & 0,05 \\
\hline Não & 20,0 & 1,00 & & 1,00 & \\
\hline Sim & 47,0 & $2,35(1,24-4,44)$ & & $1,93(1,01-3,68)$ & \\
\hline Consome frutas diariamente & & & $<0,01$ & & 0,01 \\
\hline Não & 34,7 & 1,00 & & 1,00 & \\
\hline Sim & 57,0 & $1,64(1,26-2,15)$ & & $1,42(1,08-1,86)$ & \\
\hline Consome refrigerante & & & 0,02 & & 0,60 \\
\hline Não & 51,9 & $1,40(1,06-1,83)$ & & $1,08(0,82-1,41)$ & \\
\hline Sim & 37,2 & 1,00 & & 1,00 & \\
\hline Estado nutricional & & & 0,01 & & 0,05 \\
\hline Eutrófico & 39,3 & $1,14(0,76-1,72)$ & & $1,07(0,70-1,63)$ & \\
\hline Sobrepeso & 56,9 & $1,65(1,10-2,48)$ & & $1,45(0,96-2,19)$ & \\
\hline Obeso & 34,5 & 1,00 & & 1,00 & \\
\hline Hipertensão arterial & & & $<0,01$ & & 0,01 \\
\hline Não & 39,0 & 1,00 & & 1,00 & \\
\hline Sim & 61,7 & $1,58(1,20-2,09)$ & & $1,45(1,09-1,94)$ & \\
\hline Autopercepção da saúde & & & $0,03^{*}$ & & $0,09^{*}$ \\
\hline Excelente ou muito boa & 50,4 & $1,61(0,95-2,71)$ & & $1,43(0,87-2,36)$ & \\
\hline Boa & 39,3 & $1,25(0,73-2,14)$ & & $1,20(0,72-2,32)$ & \\
\hline Regular ou ruim & 31,3 & 1,00 & & 1,00 & \\
\hline
\end{tabular}

RP: razão de prevalência; IC95\%: intervalo de 95\% confiança; *Valor p do teste de tendência linear 
Tabela 2. Associação da prática de atividade física no lazer com nível de qualidade de vida (em escore-z) dos professores da Universidade Federal do Rio Grande (FURG). Rio Grande, RS, 2015 (N=270)

\begin{tabular}{|c|c|c|c|c|c|}
\hline \multirow{2}{*}{ Variável } & Qualidade de vida & Análise bruta & \multirow{2}{*}{ Valor $\mathbf{p}$} & Análise ajustada* & \multirow{2}{*}{ Valor $\mathbf{p}$} \\
\hline & Média (DP) & Beta (IC95\%) & & Beta (IC95\%) & \\
\hline$A F \geq 150 \mathrm{~min} / \mathrm{sem}$ & & & 0,01 & & 0,04 \\
\hline Não & $-0,27(2,01)$ & 0,00 & & 0,00 & \\
\hline Sim & $0,36(1,85)$ & $0,62(0,15 ; 1,09)$ & & $0,53(0,03 ; 1,03)$ & \\
\hline AF em tercis & & & 0,001 & & 0,002 \\
\hline 1 (menos ativos) & $-0,38(1,98)$ & 0,00 & $<0,001 * *$ & 0,00 & $0,002^{* *}$ \\
\hline 2 & $-0,04(1,93)$ & $0,34(-0,17 ; 0,85)$ & & $0,22(-0,32 ; 0,75)$ & \\
\hline 3 (mais ativos) & $1,06(1,70)$ & $1,44(0,71 ; 2,17)$ & & $1,34(0,58 ; 2,10)$ & \\
\hline AF em quartis & & & 0,001 & & 0,003 \\
\hline 1 (menos ativos) & $-0,45(2,02)$ & 0,00 & $0,001^{* *}$ & 0,00 & $0,01^{* *}$ \\
\hline 2 & $0,02(1,85)$ & $0,47(-0,14 ; 1,08)$ & & $0,37(-0,25 ; 0,98)$ & \\
\hline 3 & $-0,07(1,98)$ & $0,38(-0,22 ; 0,98)$ & & $0,22(-0,41 ; 0,85)$ & \\
\hline 4 (mais ativos) & $1,06(1,70)$ & $1,51(0,76 ; 2,25)$ & & $1,39(0,62 ; 2,16)$ & \\
\hline
\end{tabular}

AF: atividade física; DP: desvio-padrão; IC $95 \%$ : intervalo de $95 \%$ de confiança; *Ajuste para faixa etária, titulação, regime de trabalho, tabagismo, consumo de frutas, hábito de tomar café da manhã, estado nutricional e hipertensão arterial; **Valor p do teste de tendência linear

Tabela 3. Associação da prática de atividade física no lazer (em tercis) com os domínios de qualidade de vida (pontuação de 0 a 100) entre os professores da Universidade Federal do Rio Grande (FURG). Rio Grande, RS, 2015 (N=270)

\begin{tabular}{|c|c|c|c|c|}
\hline \multirow{2}{*}{ Domínios de qualidade de vida } & \multicolumn{3}{|c|}{ Atividade física no lazer em tercis (média e DP) } & \multirow{2}{*}{ Valor $p$} \\
\hline & Menos ativos & Tercil do meio & Mais ativos & \\
\hline Capacidade funcional & $81,8(16,3)$ & $89,5(12,7)$ & $95,1(7,2)$ & $\begin{array}{l}<0,01^{* *} \\
<0,01^{* * *}\end{array}$ \\
\hline Aspectos físicos & $88,3(25,2)$ & $78,2(33,7)$ & $82,2(32,8)$ & $\begin{array}{l}0,05^{* * *} \\
0,10^{* * *}\end{array}$ \\
\hline Dor & $67,9(17,8)$ & $69,7(18,6)$ & $73,9(15,5)$ & $\begin{array}{l}0,23^{*} \\
0,10^{* * *}\end{array}$ \\
\hline Estado geral de saúde & $64,3(19,4)$ & $70,8(16,7)$ & $80,5(18,0)$ & $\begin{array}{l}<0,01 * \\
<0,01^{* * *}\end{array}$ \\
\hline Vitalidade & $55,2(20,9)$ & $60,1(20,1)$ & $72,2(16,3)$ & $\begin{array}{l}<0,01^{*} \\
<0,01^{* * *}\end{array}$ \\
\hline Aspectos sociais & $74,2(24,7)$ & $75,0(21,1)$ & $82,2(17,1)$ & $\begin{array}{l}0,21^{* * *} \\
0,10^{* * *}\end{array}$ \\
\hline Aspectos emocionais & $71,9(37,0)$ & $68,6(36,8)$ & $82,5(29,8)$ & $\begin{array}{l}0,11^{*} \\
0,32^{* * * *}\end{array}$ \\
\hline Saúde mental & $68,6(19,3)$ & $71,3(18,5)$ & $79,2(13,7)$ & $\begin{array}{c}0,01^{* *} \\
<0,01^{* * *}\end{array}$ \\
\hline
\end{tabular}

DP: desvio-padrão; *Análise de variância (ANOVA); **Teste Kruskal-Wallis; ***Teste de tendência linear

\section{DISCUSSÃO}

Este estudo teve como objetivo investigar a prática de atividade física e sua associação com qualidade de vida em professores universitários. A prevalência de atividade física encontrada (43\%; IC95\%: 37 a 49) foi semelhante à de um estudo feito em 2008 com 293 professores da Universidade Estadual de Maringá, mostrando que 43,4\% dos professores foram classificados como ativos ou muito ativos ${ }^{14}$. Essa prevalência não variou conforme situação conjugal, 
tempo de carreira nem instituto, assim como no presente trabalho. Já um estudo conduzido em 2010, com 24 docentes do curso superior de Educação Física da Universidade Federal de Pelotas, apontou que $66,7 \%$ foram considerados suficiente ativos no lazer ${ }^{15}$. Provavelmente essa prevalência tenha sido mais elevada devido à área de atuação destes professores ser direcionada à atividade física.

Em relação aos fatores associados, verificou-se que professores com idade mais avançada foram mais ativos no lazer, diferentemente do que aponta a literatura ${ }^{16}$. Atribui-se essa divergência às particularidades da profissão do professor, que precisa de alguns anos para consolidar sua carreira e conseguir encontrar mais tempo para o lazer. Supõe-se também que professores com idades mais avançadas se preocupem mais com sua saúde, contribuindo para a adoção de um estilo de vida fisicamente ativo. Verificou-se associação entre o consumo regular de frutas e o hábito de tomar café da manhã com maior prevalência de atividade física no lazer. Estudos mostraram que hábitos saudáveis podem induzir a outros comportamentos benéficos à saúde, havendo um efeito de clustering entre os fatores comportamentais ${ }^{17,18}$.

No que se refere à qualidade de vida, observou-se que professores fisicamente ativos atingiram maior escore para este desfecho em saúde, demonstrando gradiente dose-resposta significativo. Com relação aos mecanismos biológicos pelos quais a atividade física atua melhorando a qualidade de vida, acreditamos ser uma relação mediada pelos efeitos da atividade física sobre a aptidão muscular e cardiorrespiratória, composição corporal, proteção contra doenças crônicas e depressão ${ }^{10}$.

Um estudo feito com professores, funcionários e alunos da Universidade Católica de Pelotas indicou que os indivíduos mais ativos fisicamente tiveram maior nível de qualidade de vida ${ }^{19}$. Outro estudo, feito com funcionários da Universidade Estadual de Londrina, com indivíduos entre 60 e 70 anos, apontou que a qualidade de vida esteve associada ao maior nível de atividade física ${ }^{20}$.

Tratando-se da associação da atividade física com cada domínio de qualidade de vida, uma pesquisa conduzida com funcionários públicos da Universidade Estadual do Piauí, usando o SF-36, mostrou que indivíduos mais ativos atingiram maior pontuação nos domínios capacidade funcional e saúde mental ${ }^{21}$. Em estudo de base populacional com adultos da zona urbana de Montes Claros (Minas Gerais), os autores verificaram maior escore de qualidade de vida para os indivíduos fisicamente ativos nos seguintes domínios: capacidade funcional ( $p=0,01)$, aspectos físicos $(p=0,02)$, vitalidade $(p=0,05)$ e aspectos emocionais $(p=0,04)^{22}$.

Ainda sobre os domínios de qualidade de vida, estudo de revisão sistemática, com 38 artigos publicados entre 1980 e 2010, concluiu que os domínios mais consistentemente associados à atividade física foram: funcional (em 100\% dos estudos), vitalidade e saúde mental (em 80\% dos estudos) ${ }^{4}$. O domínio social não mostrou associação (apenas um estudo encontrou) e, para o domínio dor, a associação ficou inconclusiva, apesar de a maioria dos estudos não ter encontrado associação 4 .

No presente estudo, apenas os domínios dor, aspectos sociais e aspectos emocionais não apresentaram associação estatisticamente significativa com a prática de atividade física. Salienta-se que, no domínio aspectos físicos, os indivíduos menos ativos alcançaram maior escore de qualidade de vida. Esse mesmo achado foi obtido em um estudo experimental randomizado, com mulheres saudáveis entre 40 e 74 anos da Nova Zelândia, que apontou redução na pontuação do domínio físico para o grupo intervenção ${ }^{23}$. Os autores atribuem esse resultado a um possível aumento no número de quedas e lesões no grupo fisicamente ativo.

Como limitações, citam-se a baixa taxa de resposta (40\%) e o tipo de estudo (transversal). Quanto à taxa de resposta, o método empregado de questionários autoaplicáveis pode ter explicado, em parte, a baixa adesão. Estudo de revisão sistemática, envolvendo artigos sobre qualidade de vida em professores brasileiros, mostrou que a taxa de resposta foi inferior a $50 \%$ em todos os estudos cuja amostra não tenha sido por conveniência ${ }^{5}$. Acredita-se que os professores que não tenham preenchido o questionário tenham um perfil menos saudável, podendo apresentar menores níveis de atividade física e menor escore de qualidade vida, o que agiria subestimando as associações reportadas no presente estudo. 
Vale salientar que a baixa taxa de resposta pode ter prejudicado também o poder (precisão) do estudo. Por ter sido uma pesquisa censitária, não foi necessário cálculo de tamanho amostral. Cabe, no entanto, destacar que foi possível detectar, como estatisticamente significativas, razões de prevalência (RP) maiores do que 1,4, bem como coeficientes beta acima de 0,5 escores-ze diferenças no escore de qualidade de vida de pelo menos 10 pontos entre os terços de nível de atividade física.

Sobre o delineamento do estudo, como todas as variáveis foram coletadas no mesmo momento, sabe-se que algumas delas podem ter sido afetadas pelo viés de causalidade reversa. Por exemplo, pode ser que a prevalência de atividade física tenha sido maior para os indivíduos hipertensos por eles terem se tornado ativos em decorrência da sua condição de saúde. O mesmo pode ter acontecido para aqueles indivíduos com sobrepeso e os ex-fumantes.

Este estudo pode trazer algumas implicações práticas. Primeiro, observou-se que professores que apresentam um comportamento saudável tendem também a apresentar outros, denotando maior preocupação e cuidado com a sua saúde (o que se conhece como efeito de "cluster"). Logo, se se conseguir fazer com que os professores se tornem fisicamente ativos, isso poderá repercutir positivamente sobre outros comportamentos saudáveis.

Segundo, ao se demonstrar que o nível de atividade física esteve associado, de forma diretamente proporcional, ao escore de qualidade de vida, cria-se mais um argumento em favor da promoção e incentivo à prática de atividade física no ambiente universitário. Seria também uma forma interessante de integrar e aproximar os professores dos alunos e criar uma rede de apoio social.

Com os dados levantados, conclui-se que os professores devem dar mais atenção a ter um estilo de vida saudável. Menos da metade dos professores foram considerados fisicamente ativos no tempo de lazer. Além disso, demonstrou-se que, quanto maior o nível de atividade física de lazer, maiores foram os escores de qualidade de vida. Essa associação ocorreu principalmente para os seguintes domínios: capacidade funcional, estado geral de saúde e vitalidade. Portanto, intervenções, sejam no âmbito universitário ou comunitário, visando à promoção da prática de atividade física, poderão repercutir em melhorias à qualidade de vida deste público.

\section{AGRADECIMENTOS}

SCD é bolsista de produtividade em pesquisa do CNPQ.

\section{REFERÊNCIAS}

1. Landeiro GMB, Pedrozo CCR, Gomes MJ, Oliveira ERA. Systematic review of studies on quality of life indexed on the SciELO database. Cien Saude Colet. 2011 out;16(10):4257-66. http://dx.doi.org/10.1590/ S1413-81232011001100031. PMid:22031154.

2. Dankel SJ, Loenneke JP, Loprinzi PD. Physical activity and diet on quality of life and mortality: The importance of meeting one specific or both behaviors. Int J Cardiol. 2016;202:328-30. http://dx.doi. org/10.1016/j.ijcard.2015.09.019. PMid:26422022.

3. Bize R, Johnson JA, Plotnikoff RC. Physical activity level and health-related quality of life in the general adult population: a systematic review. Prev Med. 2007;45(6):401-15. http://dx.doi.org/10.1016/j. ypmed.2007.07.017. PMid:17707498.

4. Pucci GCMF, Rech CR, Fermino RC, Reis RS. Association between physical activity and quality of life in adults. Rev Saude Publica. 2012 fev;46(1):166-79. http://dx.doi.org/10.1590/S0034-89102012000100021. PMid:22249758.

5. Davoglio TR, Lettnin CC, Baldissera CG. Avaliação da qualidade de vida em docentes brasileiros: uma revisão sistemática. Pro-Posições. 2015;26(3):145-66. http://dx.doi.org/10.1590/0103-7307201507807.

6. Ciconelli RM, Ferraz MB, Santos W, Meinão I, Quaresma MR. Tradução para a língua portugues e validação do questionário genérico de avaliação de qualidade de vida SF-36 (Brasil SF-36). Rev Bras Reumatol. 1999;39(3):144-50

7. Ciconelli RM. Medidas de avaliação de qualidade de vida. Rev Bras Reumatol. 2003;43(2):9-13.

8. DeVellis RF. Scale development: Theory and applications. Los Angeles: Sage; 2012. 
9. Brasil. Vigitel Brasil 2016: vigilância de fatores de risco e proteção para doenças crônicas por inquérito telefônico: estimativas sobre frequência e distribuição sociodemográfica de fatores de risco e proteção para doenças crônicas nas capitais dos 26 estados brasileiros e no Distrito Federal em 2016. Brasília: Ministério da Saúde; 2017.

10. World Health Organization. Global Recommendations on Physical Activity for Health. Switzerland: WHO; 2010.

11. Barros AJ, Hirakata VN. Alternatives for logistic regression in cross-sectional studies: an empirical comparison of models that directly estimate the prevalence ratio. BMC Med Res Methodol. 2003;3(1):21. http://dx.doi.org/10.1186/1471-2288-3-21. PMid:14567763.

12. Dumith SC. Proposta de um modelo teórico para a adoção da prática de atividade física. Rev Bras Ativ Fis Saude. 2008;13(2):52-62.

13. Croux C, Haesbroeck G. Principal component analysis based on robust estimators of the covariance or correlation matrix: influence functions and efficiencies. Biometrika Trust. 2000;87(3):603-18. http://dx.doi. org/10.1093/biomet/87.3.603.

14. Oliveira-Filho A, Netto-Oliveira ER, Oliveira AAB. Qualidade de vida e fatores de risco de professores universitários. Rev Educ Fis UEM. 2012;23(1):57-67. http://dx.doi.org/10.4025/reveducfis.v23i1.10468.

15. Hartwig CP, Rombaldi AJ, Silva MC. Indicadores do estilo de vida dos docentes de um curso de Educação Física. Pensar Prát. 2012;15(2):411-27. http://dx.doi.org/10.5216/rpp.v15i2.14004.

16. Dumith SC. Physical activity in Brazil: a systematic review. Cad Saude Publica. 2009;25(Supl Suppl 3):S41526. http://dx.doi.org/10.1590/S0102-311X2009001500007. PMid:20027389.

17. Dumith SC, Muniz LC, Tassitano RM, Hallal PC, Menezes AM. Clustering of risk factors for chronic diseases among adolescents from Southern Brazil. Prev Med. 2012;54(6):393-6. http://dx.doi.org/10.1016/j. ypmed.2012.03.014. PMid:22484392.

18. Tassitano RM, Dumith SC, Chica DA, Tenorio MC. Aggregation of the four main risk factors to noncommunicable diseases among adolescents. Rev Bras Epidemiol. 2014;17(2):465-78. http://dx.doi. org/10.1590/1809-4503201400020014ENG. PMid:24918417.

19. Silva RS, Silva I, Silva RA, Souza L, Tomasi E. Physical activity and quality of life. Cien Saude Colet. 2010 jan;15(1):115-20. http://dx.doi.org/10.1590/S1413-81232010000100017. PMid:20169238.

20. Porto DB, Guedes DP, Fernandes RA, Reichert FF. Perceived quality of life and physical activity in Brazilian older adults. Motricidade. 2012;8(1):33-41.

21. Costa VB, Soares NIS, Costa JES, Leone ID, Silva VF, Costa DCCO, et al. Nível de atividade física e qualidade de vida de funcionários públicos administrativos. Biomotriz. 2014;8(1):91-104.

22. Campos M, Maciel M, Rodrigues J No. Atividade física insuficiente: fatores associados e qualidade de vida. Rev Bras Ativ Fis Saude. 2012;17(6):562-72. http://dx.doi.org/10.12820/2317-1634.2012v17n6p562.

23. Lawton BA, Rose SB, Elley CR, Dowell AC, Fenton A, Moyes SA. Exercise on prescription for women aged 4074 recruited through primary care: two year randomised controlled trial. Br J Sports Med. 2009;43(2):120-3. PMid:19204077. 


\section{Errata}

No artigo "ATIVIDADE FÍSICA E QUALIDADE DE VIDA DE PROFESSORES UNIVERSITÁRIOS", DOI https://doi.org/10.1590/1414-462X202028030593, publicado no periódico Cadernos Saúde Coletiva, vol.28, no. 3, pp. 438-446, na página 438 e no cabeçalho das páginas pares seguintes:

Onde se lia:

"Aprovado em: Dez. 02, 2019"

Leia-se:

"Aprovado em: Out. 02, 2019"

\section{(c) (1)}

Este é um artigo publicado em acesso aberto (Open Access) sob a licença Creative Commons Attribution, que permite uso, distribuição e reprodução em qualquer meio, sem restrições desde que o trabalho original seja corretamente citado. 